\title{
HALL-HIGMAN TYPE THEOREMS. II
}

\author{
BY
}

\author{
T. R. BERGER(1)
}

\begin{abstract}
This paper continues the investigations of this series. Suppose $\mathrm{K}=\mathrm{GF}(r)$ is a field for a prime $r ; G$ is a nilpotent; $V$ is a nonsingular symplectic space with form $g$; and $V$ is a faithful irreducible $\mathrm{K}[G]$-module where $G$ fixes the form $g$. This paper describes completely the structure of $G$ and its representation upon $V$ when $G$ is symplectic primitive. This latter condition is described in $\S 4$ and is a primitivity condition.
\end{abstract}

Let $C$ be a cyclic $p$-group for a prime $p$ and $Q$ an extraspecial $q$-group for a different prime $q$. Suppose $C$ acts faithfully as automorphisms of $Q$ centralizing $Z(Q)$. Assume also that $Q / Z(Q)$ is a chief factor of the semidirect product $C Q$. The representation theory of $C Q$ is well known. It was first studied thoroughly in Theorem B of Hall and Higman [10]. In this sequence of papers we look at the basic configurations arising out of Theorem B.

In Hall-Higman Type Theorems. I [2] (henceforth referred to as [I]) two questions related to Theorem B were posed. The second question will concern us here. Suppose $A G$ is a group with normal solvable subgroup $G$ and nilpotent complement $A$. Assume that $S \leqslant G$ is an extraspecial s-subgroup normalized by $A G$ where $(|A|, s)=1$. Further, assume that $Z(S) \leqslant Z(A G)$, $S / Z(S)$ is an $A G$-chief factor, and $A$ is faithful upon $S$. Let $\mathbf{k}$ be a field of characteristic other than $s$; and let $V$ be an irreducible $\mathrm{k}[A G]$-module nontrivial for $Z(S)$. When does $\eta_{A}$ contain a regular direct summand?

With $A=C$ and $G=S=Q$ this is just the case described in the first paragraph. The possible theorems which give answers to this question can be proved by induction in several steps. First one reduces to the case where $\mathbf{k}$ is algebraically closed and $\left.V\right|_{S}$ is irreducible. Next one is forced to look at the representation of $A G$ upon $\bar{S}=S / Z(S)$. The module $\bar{S}$ is analyzed by group-theoretic induction. That is, $\bar{S}$ is induced by a certain minimal submodule, $\vec{S}_{\mathbf{0}}$. In providing answers to our question, then, one must take into

Received by the editors October 24, 1972.

AMS (MOS) subject classifications (1970). Primary 20C15, 20C20; Secondary 16A64, $20 \mathrm{H} 20$.

Key words and phrases. Hall-Higman Theorem B, representation theory, group theory, minimal module, symplectic primitive module.

(1) Research partially supported by NSF Grant GP 38879. 
account the structure of $\bar{S}_{0}$ and the induction from $\bar{S}_{0}$ to $\bar{S}$. The whole method being sketched here is fully described in $[4, \S 7]$. In this paper we are concerned with the structure of $\bar{S}_{0}$. In fact, $\bar{S}_{0}=S_{0} / Z(S)$ where $S_{0}$ is an extraspecial subgroup of $S$. Further, the stabilizer of $\bar{S}_{0}$ is $N_{A G}\left(S_{0}\right)=$ $A_{0} G_{0}$ where $A_{0} \leqslant A$ and $G_{0} \leqslant G$. Further, $\bar{S}_{0}$ has certain minimality conditions imposed upon it. We describe these now.

The commutator map induces a nonsingular symplectic form upon $\bar{S}_{0}$ fixed by $A_{0} G_{0}$. Further, things are so chosen that one of the following two conditions holds.

(1) $\left.\bar{S}_{0}\right|_{L}$ is homogeneous for all $L$ normal in $A_{0} G_{0}$ (i.e. $\bar{S}_{0}$ is quasiprimitive).

(2) If $L$ is normal in $A_{0} G_{0}$ and $\left.\bar{S}_{0}\right|_{L}=W_{1} \dot{+} \cdots+W_{t}$ where $t>1$ and the $W_{i}$ are homogeneous components then

(a) $t=2$;

(b) $W_{i}$ is totally isotropic; and

(c) if $M$ is normal in $A_{0} G_{0}$ and stabilizes $W_{1}$ then $\left.W_{1}\right|_{M}$ is homogeneous.

The module $\bar{S}_{0}$ satisfying these conditions is called a minimal module. The simplest configuration would be the case where $C_{A_{0} G_{0}}\left(\bar{S}_{0}\right)$ contains $G_{0}$. Therefore $G_{0}$ is in the kernel of the action of $A_{0} G_{0}$ upon $\bar{S}_{0}$. That is, what are the minimal modules over $\mathrm{GF}(s)$ for nilpotent groups?

This very special question is the topic of this paper. We shall obtain the answer for slightly more general groups in (3.10) and (3.17). By specializing, we obtain the answers desired in (3.20). We obtain a complete classification of all minimal modules for nilpotent groups. The actual groups and modules obtained are subgroups of a group $B$ described in (2.1)-(2.4). Basically, the nilpotent group and module can be of four types:

(i) a cyclic group;

(ii) and (iii) quaternion groups with two possible module actions; or

(iv) the direct product of an odd cyclic group with a 2-group which is semidihedral, dihedral, or quaternion.

The actual groups are somewhat complicated to describe. They are constructed in $\S 2$. The nilpotent groups are then subgroups of the group $B$ which act upon the module as a minimal module.

As an analogy, consider group modules over the complex field for a moment. Every module is induced by a primitive module. But primitive modules are hard to spot. A larger but more easily known class is the class of quasiprimitive modules, that is, modules which are homogeneous on every normal subgroup. 
The module $\bar{S}_{0}$ considered before corresponds to a primitive module in that it is not induced from any proper nonsingular submodule in a form-preserving manner. Such a module may not be primitive, but it is very close. We call it symplectic primitive. The definition of minimal module is the one corresponding to what we might (but do not) call symplectic quasiprimitive. Thus the symplectic primitive modules are among those we classify. $\S 4$ is an added footnote to describe certain properties of symplectic primitive modules.

Much more information on symplectic primitive and minimal modules of a general nature is contained in [4].

A few comments may be in order on how and why this particular problem arose. I was considering a solvable group $G$ and a relatively prime order nilpotent group of operators $A$ on $G$. If $A$ is fixed point free upon $G$ (i.e. $\left.C_{G}(A)=1\right)$, then is the Fitting height of $G$ bounded above by the composition length of $A$ ? I have been able to prove that the answer is yes if $A$ is $\mathbf{Z}_{p} \sim \mathbf{Z}_{p}$-free. An attack upon this question quickly reduces to a problem of representation theory [1], [6]. In fact, one is quickly led to the configurations of Theorem B of Hall and Higman. It was the fixed point free problem which led me into this area.

It quickly became apparent that the method transcended the problem. For example, the methods of this series have led to results on character degrees [7], on primitive solvable groups [8], and on 2-length [9]. Contained in the paper of Hall and Higman are the seeds of a very complete theory for the representation of solvable groups over any field. An outline of this theory is given in [4]. This paper is confined to the "very lowest level" analysis. That is, the analysis necessary to settle the fixed point free question for $\mathbf{Z}_{p} \sim \mathbf{Z}_{p}$-free operator groups. However, I have done this in such a framework that more general questions can be settled as they arise. The necessary Hall-Higman theory to prove the $\mathbf{Z}_{p} \sim \mathbf{Z}_{p}$-free theorem is already much less than is contained here. It is like the case of the cart which is motor driven and therefore capable of carrying the horse.

In a line, I consider the Hall-Higman analysis to be far more important than the fixed point free question.

In this paper we study the $A_{0}$-module $\bar{S}_{0}$. In [3] we study the character theory of $A_{0} S_{0}$, that is, the ordinary representation theory. The modular theory for $A_{0} S_{0}$ may be found in [5].

1. Preliminary remarks. All our groups are solvable. Notation is standard. For example $\mathbf{K}^{+}$denotes the additive group of a field, $\mathbf{K}$, and $\mathbf{K}^{\times}$the multiplicative group. The groups of $\S 2$ with the exception of (2.4) seem to be more or less known. 
We shall need certain preliminary results stated below.

(1.1) $[2,(2.3)]$ Suppose that $G=A N$ is a group with normal cyclic subgroup $N$ and nilpotent complement $A$ where $A \cap N=1$. If every normal abelain subgroup of $G$ is cyclic then $G$ contains a cyclic self-centralizing normal subgroup $M \geqslant N$. Further,

(a) $C_{G}(N)=\left(A \cap C_{G}(N)\right) \times N$,

(b) $\left(\left|A \cap C_{G}(N)\right|,|N|\right)=1$,

(c) $M=C_{G}(N)$ or $\left[C_{G}(N): M\right]=2$ and an $S_{2}$-subgroup of $C_{G}(N)$ is quaternion, dihedral, or semidihedral.

We remark here that by quaternion we mean quaternion of order 8 or generalized quaternion.

We introduce some notation to state the next result. Let $\mathbf{k}$ be a finite field and $\hat{\mathbf{k}}$ a finite extension of $\mathbf{k}$. Suppose $G$ is the Galois group of $\hat{\mathbf{k}} / k$, we may form the semidirect product $G \mathbf{k}^{\dot{x}}$. We call this group $T(\hat{\mathbf{k}} / \mathbf{k})$. If $\sigma x \in T(\hat{k} / \mathbf{k})$ where $\sigma \in G, x \in \hat{\mathbf{k}}^{\times}$, and if $v \in \hat{\mathbf{k}}^{+}$then $T(\hat{\mathbf{k}} / \mathbf{k})$ acts naturally upon the $\mathbf{k}$-vector space $\hat{\mathbf{k}}^{+}$by $\sigma x \cdot v=\sigma(x v)$.

(1.2) $[2,(2.5)]$ Suppose $G$ is a group with cyclic self-centralizing normal subgroup $M$. Assume that $\mathbf{k}$ is a finite field, $V$ is a faithful irreducible $\mathbf{k}[G]$-module, and $\eta_{M}$ is homogeneous. Let $\tilde{\mathbf{k}}$ be an extension of $\mathbf{k}$ by a primitive $|M| t h$ root of unity. Then there is a subfield $\hat{\mathbf{k}}$ and monomorphisms $\varphi: G \rightarrow T \widetilde{\mathbf{k}} / \hat{\mathbf{k}}$ ) and $\Phi: V \rightarrow \widetilde{\mathbf{k}}+$ (an isomorphism) so that

(a) $\varphi(x) \Phi(v)=\Phi(x v)$ all $x \in G, v \in V$;

(b) if $M=\langle x\rangle$ and $\varphi(x)=\omega$ then $\mathrm{k}(\omega)=\tilde{\mathbf{k}}$;

(c) $\varphi(G) \tilde{\mathbf{k}}^{\times}=T(\tilde{\mathrm{k}} / \hat{\mathrm{k}}) ;$ and

(d) $\varphi(G) \cap \tilde{\mathbf{k}}^{\times}=\varphi(M)$.

The hypotheses used here are slightly weaker than those of $[2,(2.5)]$. However, those stated here are the ones actually used in the proof.

Actually, there is a good deal of flexibility built into the maps $\varphi$ and $\Phi$. We state this as follows.

(1.3) Consider the situation of (1.2). Let $\gamma \in \tilde{\mathbf{k}}^{\times}$. Define $\left.\theta: T \tilde{\mathbf{k}} / \hat{\mathbf{k}}\right) \rightarrow$ $T(\tilde{\mathbf{k}} / \mathbf{k})$ and $\Theta: \tilde{\mathbf{k}}^{+} \rightarrow \tilde{\mathbf{k}}^{+}$by setting

(i) $\theta(\sigma x)=\sigma x \gamma^{\sigma} \gamma^{-1}$ for $\sigma \in G, x \in \tilde{\mathbf{k}}^{\times}$; and

(ii) $\Theta(v)=v \gamma$ for $v \in \tilde{\mathbf{k}}^{+}$.

Then $\theta, \Theta$ are isomorphisms. Further, $\varphi^{\prime}=\theta \varphi$ and $\Phi^{\prime}=\Theta \Phi$ satisfy (a)-(d) of (1.2). 
The maps $\theta, \Theta$ are easily shown to be isomorphisms. Let $\sigma \in G, x \in$ $\widetilde{\mathbf{k}}^{\times}$, and $v \in \tilde{\mathbf{k}}^{+}$. We prove that

$$
\begin{aligned}
\theta(\sigma x) \cdot \Theta(v) & =\Theta(\sigma x \cdot v), \\
\theta(\sigma x) \cdot \Theta(v) & =\left[\sigma x \gamma^{\sigma} \gamma^{-1}\right] \cdot[v \gamma]=\sigma\left(x \gamma^{\sigma} \gamma^{-1} v \gamma\right) \\
& =\sigma(x v) \gamma=\Theta(\sigma x \cdot v) .
\end{aligned}
$$

The rest of the proof follows directly from this by computation.

This is all we shall need in the way of preliminary results.

2. Some examples. In this section we will consider certain examples of groups and actions. These groups and actions are the critical cases in the theorems that follow. There are four actions in all. Each is indexed by a number. In the later parts of this paper we shall index the whole description by these numbers.

We fix the following notation.

$r$ a prime.

$m>0$ an integer.

$\mathbf{K}=\mathbf{G F}(r)$.

$\widetilde{\mathbf{K}}=\mathbf{G F}\left(r^{m}\right)$.

$\hat{\mathbf{K}}=\mathrm{GF}\left(r^{2 m}\right)$.

In each case, we describe a group $G$ with certain subgroups $B$ and $C$, a $\mathrm{K}[G]$-module $V$, and a nonsingular symplectic form $g$ upon $V$ which is fixed by $G(g(u, v)=g(x u, x v)$ all $u, v \in V ; x \in G)$.

(2.1) $G$ is cyclic of order $r^{m}+1$.

Let $V=\hat{\mathbf{K}}^{+}$and $\varphi: \epsilon \rightarrow \epsilon^{r^{m}}$, the automorphism of order two of $\hat{\mathbf{K}}$. Let $G$ be the multiplicative subgroup of $\hat{\mathbf{K}}^{x}$ of order $r^{m}+1$. Then $G$ acts upon $V$ by multiplication.

Let $\operatorname{Tr}: \widetilde{\mathbf{K}} \rightarrow \mathbf{K}$ be the trace map. Choose $\mu \in \hat{\mathbf{K}}^{\times}$so that $\mu^{\varphi}=-\mu$ (if $r=2$ let $\mu=1$ ) and set

$$
g(u, v)=\operatorname{Tr}\left(\mu\left(u v^{\varphi}-u^{\varphi} v\right)\right) ; \quad u, v \in V .
$$

We set $G=B$ and $C=1$.

$$
r^{m} \equiv-1(\bmod 4), \quad|G|=2\left(r^{m}+1\right), \quad r>2 .
$$

Consider the space $V$ and form $g$ of Example (2.1). Let $Q$ be the 2-Sylow subgroup and $C$ the Hall-complement to $Q$ in $G$ of (2.1). Choose $\nu \in \hat{\mathbf{K}}^{\mathrm{X}}$ so that $\nu^{r^{m}+1}=-1$. Form the semidirect product $\langle\varphi\rangle \hat{\mathbf{K}} \times$ where $\varphi: \epsilon \rightarrow \epsilon^{r^{m}}$ is the automorphism of order two. In this group we take

$$
B=\langle Q, \varphi \nu\rangle \text { and } G=C B \text {. }
$$


Then $B$ is quaternion of order $2^{t+1}$ where $2^{t} \mid t+1$. Further, $C$ is a cyclic normal subgroup of $G$ inverted by $\varphi v$ of odd order $\left(r^{m}+1\right) / 2^{t}$. Finally; $C Q=C \times Q$ is a direct product.

All elements $\nu$ such that $\nu^{r^{m}+\alpha}=-1$ lie in $\nu Q C$. So any such choice $\nu^{\prime}$ satisfies $\varphi \nu^{\prime} \in G$. The group $G$ is independent of the choice of $\nu$.

$$
r^{m} \equiv 1(\bmod 4), \quad|G|=2\left(r^{m}-1\right), \quad r>2 .
$$

Let $V$ be a 2-dimensional $\widetilde{\mathbf{K}}$-space with basis $e_{1}, e_{2}$. So $V=\widetilde{\mathbf{K}} e_{1} \dot{+} \widetilde{\mathbf{K}}_{2}$ is a $2 m$-dimensional $\mathrm{K}$-space. Let $Q_{0}=\langle\nu\rangle$ and $C_{0}=\langle\eta\rangle$ be, respectively, the 2-Sylow subgroup and the Hall $2^{\prime}$-subgroup of $\widetilde{\mathbf{K}}^{\times}$. Using matrices to denote linear transformations written in the basis $e_{1}, e_{2}$, let

$$
Q=\left\langle\left[\begin{array}{cc}
\nu & \\
& \nu^{-1}
\end{array}\right]\right\rangle, \quad C=\left\langle\left[\begin{array}{cc}
\eta & \\
& \eta^{-1}
\end{array}\right]\right\rangle \text { and } \tau=\left[\begin{array}{c}
-1 \\
1
\end{array}\right] .
$$

Set $B=\langle Q, \tau\rangle$ and $G=C B$. Then $B$ is quaternion of order $2^{t+1}$ where $2^{t}|| r^{m}-1$. Further, $C$ is a cyclic normal subgroup of $G$ inverted by $\tau$ of odd order $\left(r^{m}-1\right) / 2^{t}$. Finally, $C Q=C \times Q$ is a direct product.

If $u=\alpha e_{1}+\beta e_{2}$ and $v=\alpha^{\prime} e_{1}+\beta^{\prime} e_{2}$ where $\alpha, \alpha^{\prime}, \beta, \beta^{\prime} \in \widetilde{\mathbf{K}}$ then set $g^{*}(u, v)=\alpha \beta^{\prime}-\alpha^{\prime} \beta$. Let $\operatorname{Tr}: \widetilde{\mathbf{K}} \rightarrow \mathbf{K}$ be the trace map and set $g(u, v)=$ $\operatorname{Tr}\left(g^{*}(u, v)\right)$.

$$
m=2 n, \quad r^{n} \equiv 1(\bmod 4), \quad|G|=2\left(r^{m}-1\right), \quad r>2 .
$$

Let $V$ be a 2 -dimensional $\widetilde{\mathrm{K}}$-space with basis $e_{1}, e_{2}$. So $V$ is a $2 m$ dimensional K-space. Let $\varphi: \epsilon \rightarrow \epsilon^{r n}$ be the automorphism of order two of $\widetilde{\mathbf{K}}$. Let $Q_{0}=\langle\nu\rangle$ be the 2-Sylow subgroup of $\widetilde{\mathbf{K}}^{\times} ; D_{0}=\langle\theta\rangle$ the subgroup of order $\left(r^{n}+1\right) / 2$ in $\widetilde{\mathbf{K}}^{\times}$; and $C_{0}=\langle\eta\rangle$ the Hall-complement to $Q_{0} C_{0}$ in $\widetilde{\mathbf{K}}^{\times}$. Using matrices again to denote linear transformations in the basis $e_{1}, e_{2}$ we set

$$
Q=\left\langle\left[\begin{array}{cc}
\nu & \\
& \nu^{-1}
\end{array}\right]\right\rangle, D=\left\langle\left[\begin{array}{cc}
\theta & \\
& \theta^{-1}
\end{array}\right]\right\rangle \text { and } C=\left\langle\left[\begin{array}{cc}
\eta & \\
& \eta^{-1}
\end{array}\right]\right\rangle \text {. }
$$

Set $\tau=\left[L_{\varphi}^{\varphi}\right]$ where $\tau\left(\alpha e_{1}+\beta e_{2}\right)=\beta^{\varphi} e_{1}-\alpha^{\varphi} e_{2} ; \alpha, \beta \in \widetilde{\mathbf{K}}$. Then the group $G=\langle C, D, Q, \tau\rangle$ has a semidihedral 2-Sylow subgroup $S=\langle Q, \tau\rangle$ of order $2^{t+2}$ where $2^{t} \mid r^{n}-1$; a nilpotent subgroup $B=S \times D$ where $|D|=\left(r^{n}+1\right) / 2$; and a normal cyclic subgroup $C$ of odd order $\left(r^{n}-1\right) / 2^{t}$ which is inverted by $\tau$.

For $u=\alpha e_{1}+\beta e_{2}$ and $v=\alpha^{\prime} e_{1}+\beta^{\prime} e_{2}$ where $\alpha, \alpha^{\prime}, \beta, \beta^{\prime} \in \widetilde{\mathbf{K}}$ set $g^{*}(u, v)=\beta \alpha^{\prime}-\alpha \beta^{\prime}$. Let $\operatorname{Tr}: \widetilde{\mathbf{K}} \rightarrow \mathbf{K}$ be the trace map and set $g(u, v)=$ $\operatorname{Tr}\left(g^{*}(u, v)\right)$. 
3. Some minimal $\mathrm{K}[G]$-modules. Suppose $H$ is a group; $\mathbf{k}$ is a field; $U$ is an irreducible $\mathbf{k}[H]$-module; and $g$ is a nonsingular symplectic form upon $U$ fixed by $H$. We say that $U$ is a minimal $\mathbf{k}[H]$-module provided one of the following occurs.

(1) $U l_{N}$ is homogeneous for all normal subgroups $N \leqslant H$; or

(2) for any normal subgroup $N \leqslant H$ such that $\left.U\right|_{N} \cong U_{1}+\cdots+U_{t}$ where $t>1$ and the $U_{i}$ are homogeneous components then

(i) $t=2$;

(ii) $U_{i}$ is totally isotropic for $i=1,2$; and

(iii) $\left.U_{1}\right|_{L}$ is homogeneous for all normal subgroups $L$ of $H$ contained in the stabilizer in $H$ of $U_{1}$.

More information on general minimal modules is contained in [4]. In this section we wish to classify minimal modules for, nilpotent and slightly more general groups. For nilpotent groups, the answer has already been given. The groups appear as subgroups in the examples of $\S 2$. Further, the modules and forms are given there too.

The analysis breaks into two parts depending upon the two parts of the definition for minimal module. We shall fix the following hypotheses throughout this section.

(3.1) HYPOTHESIS. $\mathrm{K}=\mathrm{GF}(r)$ for a prime $r$.

(1) $V$ is a $2 m$-dimensional $\mathrm{K}$-vector space.

(2) $g: V \times V \rightarrow \mathbf{K}$ is a nonsingular symplectic form upon $V$.

(3) $H=A N$ is a group with normal cyclic subgroup $N$ and nilpotent complement $A$ where $A \cap N=1$.

(4) $V$ is a faithful minimal $\mathrm{K}[H]$-module where $H$ fixes the form $g$ (i.e. $g(x u, v)=g\left(u, x^{-1} v\right)$ for all $\left.x \in H ; u, v \in V\right)$.

We may first determine the structure of normal abelian subgroups.

(3.2) If $L \Delta H$ is abelian then $L$ is cyclic.

If $V_{L}$ is homogeneous then the result is obvious. So assume that $V_{L}=$ $U_{1}+\cdots+U_{t}$ where $t>1$ and the $U_{i}$ are homogeneous components. In particular, $t=2$ and $U_{1}$ and $U_{2}$ are complementary totally isotropic subspaces of $V$. If $x \in G$ and $u_{1} \in U_{1}, u_{2} \in U_{2}$, then $g\left(x u_{1}, u_{2}\right)=$ $g\left(u_{1}, x^{-1} u_{2}\right)$. From this we know that $C_{L}\left(U_{1}\right)=C_{L}\left(U_{2}\right)$. But $V$ is faithful so that $U_{1}$ is faithful. Now $U_{1}$ is a homogeneous $L$-module and $L$ is abelian implying that $L$ is cyclic.

Using (3.2) and (1.1) we know the following: 
(3.3) $H$ contains a cyclic self-centralizing normal subgroup $M \geqslant N$. Further,

(a) $C_{H}(N)=\left(A \cap C_{H}(N)\right) \times N$;

(b) $\left(\left|A \cap C_{H}(N)\right|,|N|\right)=1$; and

(c) $M=C_{H}(N)$ or $\left[C_{H}(N): M\right]=2$ and an $S_{2}$-subgroup of $C_{H}(N)$ is quaternion, dihedral, or semidihedral.

Let $\mathrm{K}^{*}$ be an extension of $\mathrm{K}$ by a primitive $|M|$ th root of unity. There are two possibilities for $V_{M}$. If $V_{M}$ is homogeneous we set $H_{0}=H \geqslant M \geqslant$ $N$ and $U=V$. If $\left.V\right|_{M}=V_{1}+V_{2}$ where the $V_{i}$ are homogeneous components we let $H_{0} \geqslant M \geqslant N$ be the stabilizer in $H$ of $V_{1}$ and $U=V_{1}$. Observe that $U$ is an irreducible $K\left[H_{0}\right]$-module and $U_{M}$ is homogeneous. Since $V_{1}$ and $V_{2}$ are contragredient $\mathrm{K}\left[H_{0}\right]$-modules, $U$ is faithful for $H_{0}$.

We may now apply (1.2) to obtain:

(3.4) There is a subfield $\mathbf{K}_{0}$ of $\mathbf{K}^{*}$ and monomorphisms $\psi: H_{0} \rightarrow$ $T\left(\mathrm{~K}^{*} / \mathrm{K}_{0}\right)$ and $\Psi: U \rightarrow \mathrm{K}^{*+}$ ( $\Psi$ is an isomorphism) so that

(a) $\psi(x) \Psi(v)=\Psi(x v)$ for all $x \in H_{0} ; v \in U$;

(b) if $M=\langle x\rangle$ and $\psi(x)=\omega$ then $\mathrm{K}^{*}=\mathrm{K}(\omega)$;

(c) $\psi\left(H_{0}\right) \mathrm{K}^{* \times}=T\left(\mathrm{~K}^{*} / \mathrm{K}_{0}\right)$; and

(d) $\psi\left(H_{0}\right) \cap \mathrm{K}^{* \times}=\psi(M)$.

Before proceeding with the argument let us introduce some new apparatus. Let $\alpha: x \rightarrow x^{-1}$ for $x \in H$. Then $\alpha$ is an antiautomorphism of $H$. We may extend $\alpha$ linearly to $K[H]$. Since $K[M]$ is commutative, $\alpha$ is actually an automorphism of this subalgebra. Since $g(x u, v)=g\left(u, x^{\alpha} v\right)$ for all $u, v \in$ $V$ and $x \in H$ we must have

$$
g(b u, v)=g\left(u, b^{\alpha} v\right) \text { for } u, v \in V ; b \in \mathbf{K}[H] .
$$

Now we are ready to treat the two separate cases.

(3.5) Hypothesis. Assume (3.1). Suppose $\eta_{M}$ is homogeneous.

In this case $U=V$ and $H_{0}=H$. Further, $\mathrm{K}^{*}=\hat{\mathrm{K}}=\mathrm{GF}\left(r^{2 m}\right)$ since $V \cong \mathrm{K}^{*+}$. There is an automorphism $\varphi: \epsilon \rightarrow \epsilon^{r^{m}}$ of order two upon $\hat{\mathbf{K}}$. $\mathrm{Then}_{i} \widetilde{\mathbf{K}}=\mathrm{GF}\left(r^{m}\right)$ is the fixed field of $\varphi$.

Let $e \in \mathrm{K}[M]$ be a primitive central idempotent such that $e V \neq(0)$. By (3.4)(b), $\left.V\right|_{M}$ is irreducible so that $e$ is actually unique. Now $g(u, v)=$ $g(e u, v)=g\left(u, e^{\alpha} v\right)$ for all $u, v \in V$. Therefore, $e^{\alpha} V \neq(0)$ and $e^{\alpha}=e$. We now know that $\alpha$ is an automorphism of $\mathrm{K}[M] e$.

We may extend $\psi$ linearly to $\mathrm{K}[M]$. Therefore, $\psi$ maps $\mathrm{K}[M] e$ isomorphically onto $\hat{\mathbf{K}}$. By this process, $\alpha$ must map to $\varphi$. We use the nota- 
tion $\psi(b)=\bar{b}$ and $\Psi(u)=\bar{u}$ for $b \in \mathbf{K}[H]$ and $u \in V$. Define

Then we have

$$
g^{*}(u, \bar{v})=g(u, v) \text { for all } u, v \in V \text {. }
$$

$$
\begin{aligned}
g^{*}(\bar{u}, \bar{v}) & =g^{*}(\bar{u} \cdot \overline{1}, \bar{v})=g^{*}\left(\overline{1}, \bar{u}^{\varphi} \bar{v}\right)=-g^{*}\left(\bar{u}^{\varphi} \bar{v}, \overline{1}\right) \\
& =-g^{*}\left(\bar{u}^{\varphi} \bar{v} \cdot \overline{1}, \overline{1}\right)=-g^{*}\left(\overline{1},\left(\bar{u}^{\varphi} \bar{v}\right)^{\varphi} \cdot \overline{1}\right)=-g^{*}\left(\overline{1}, \bar{u} \bar{v}^{\varphi}\right) .
\end{aligned}
$$

From this calculation we readily observe the following:

(3.6) (i) $g^{*}\left(\overline{1}, \bar{u}^{\varphi} \bar{v}+\bar{u} \bar{v}^{\varphi}\right)=0$;

(ii) $g(u, v)=g^{*}\left(1,2^{-1}\left(\bar{u}^{\varphi} \bar{v}-\bar{u} \bar{v}^{\varphi}\right)\right)$ if $r>2$; and

(iii) $g(u, v)=g^{*}\left(\overline{1}, \bar{u}^{\varphi} v\right)$

for all $u, v \in V$ and $\overline{1} \in \hat{\mathbf{K}}^{\times}$.

Let us first settle the case where $r=2$. By (3.6) (i) we have $g^{*}\left(1, \bar{u}^{\varphi} v\right)=$ $g^{*}\left(1, \bar{u} \bar{v}^{\varphi}\right)$. Since $g$ is nonsingular, the map $\beta \rightarrow g^{*}(1, \beta)$ is $\mathbf{K}$-linear from $\hat{\mathbf{K}}^{+}$onto $\mathbf{K}^{+}$. Therefore $g^{*}(1, \beta)=\operatorname{tr}(\delta \beta)$ for some $\delta \in \hat{\mathbf{K}}^{\times}$where $\operatorname{tr}: \hat{\mathbf{K}} \rightarrow$ $\mathbf{K}$ is the trace map. But if $\operatorname{Tr}: \widetilde{\mathbf{K}} \rightarrow \mathbf{K}$ is the trace map, then $g^{*}(1, \beta)=$ $\operatorname{Tr}\left(\delta \beta+\delta^{\varphi} \beta^{\varphi}\right)$. By our observation above we have $g^{*}(1, \beta)=g^{*}\left(1, \beta^{\varphi}\right)$. So

$$
0=g^{*}\left(\overline{1}, \beta+\beta^{\varphi}\right)=\operatorname{tr}\left(\delta\left(\beta+\beta^{\varphi}\right)\right)=\operatorname{Tr}\left(\left(\delta+\delta^{\varphi}\right)\left(\beta+\beta^{\varphi}\right)\right) \text {. }
$$

Since $\beta \in \hat{\mathbf{K}}^{+}$is arbitrary we have $\operatorname{Tr}\left(\left(\delta+\delta^{\varphi}\right) \gamma\right)=0$ for all $\gamma \in \widetilde{\mathbf{K}}^{+}$. Therefore, $\delta+\delta^{\varphi}=0$ or $\delta^{\varphi}=\delta$. So $\delta \in \widetilde{\mathbf{K}}^{\times}$.

We have shown that $g(u, v)=\operatorname{Tr}\left(\delta\left(\bar{u}^{\varphi} \bar{v}-\bar{u} \bar{v}^{\varphi}\right)\right)$ for some $\delta \in \widetilde{\mathbf{K}}^{\times}$.

We turn now to the case $r>2$.

The map $\bar{u} \rightarrow \bar{u}+\bar{u}^{\varphi}$ is the trace map from $\hat{\mathbf{K}}^{+}$onto $\widetilde{\mathbf{K}}^{+}$. With $\bar{v}=$ I then (i) shows that $g^{*}(T, \beta)=0$ for all $\beta \in \widetilde{K}^{+}$. The map $\bar{u} \rightarrow \bar{u}^{\varphi}-\bar{u}$ sends $\hat{\mathbf{K}}^{+}=\widetilde{\mathbf{K}}^{+} \cdot \bar{T}+\widetilde{\mathbf{K}}^{+} \cdot \mu^{-1}$ onto $2^{-1} \mu^{-1} \widetilde{\mathbf{K}}^{+}$where $\mu^{\varphi}=-\mu, \mu \in \hat{\mathbf{K}}^{\times}$. Since $g$ is nonsingular, we know that $\beta \rightarrow g\left(1,2^{-1} \mu^{-1} \beta\right)$ is a $K$-linear mapping of $\widetilde{\mathbf{K}}^{+}$onto $\mathbf{K}$. Therefore, there is some $\delta \in \widetilde{\mathbf{K}}^{\times}$such that $g^{*}\left(1,2^{-1} \mu^{-1} \beta\right)=$ $\operatorname{Tr}(\delta \beta)$ where $\operatorname{Tr}: \widetilde{\mathbf{K}} \rightarrow \mathbf{K}$ is the trace map. From this we have:

$$
g(u, v)=g^{*}\left(1,2^{-1} \mu^{-1}\left[\mu\left(u^{\varphi} \bar{v}-\bar{u} \bar{v}^{\varphi}\right)\right]\right)=\operatorname{Tr}\left(\delta \mu\left(\bar{u}^{\varphi} \bar{v}-\bar{u} \bar{v}^{\varphi}\right)\right)
$$

(3.7) Fix $\mu \in \hat{\mathbf{K}}^{\times}$so that $\mu^{\varphi}=-\mu$. If $r=2$ let $\mu=1$. Then, for some $\delta \in \widetilde{\mathbf{K}}^{\times}, g(u, v)=\operatorname{Tr}\left(\delta \mu\left(\bar{u}^{\varphi} \bar{v}-\bar{u} \bar{v}^{\varphi}\right)\right)$ for all $u, v \in V$ and $\Psi(u)=\bar{u}$.

We now alter $\psi$ and $\Psi$ so that $\delta=1$. The norm map $\bar{u} \rightarrow \bar{u} \bar{u}^{\varphi}$ takes $\hat{\mathbf{K}}^{\times}$ onto $\widetilde{\mathbf{K}}^{\times}$. So we may choose $\gamma \in \hat{\mathbf{K}}^{\times}$such that $\gamma \gamma^{\varphi}=\delta$. By (1.3) there are isomorphisms $\theta: T\left(\hat{\mathbf{K}} / \mathbf{K}_{0}\right) \rightarrow T\left(\hat{\mathbf{K}} / \mathbf{K}_{0}\right)$ and $\Theta: \hat{\mathbf{K}}^{+} \rightarrow \hat{\mathbf{K}}^{+}$so that $\psi^{\prime}=\theta \psi$ and $\Psi^{\prime}=\Theta \Psi$ satisfy all the conditions of (3.4). In addition, $\Theta(u)=\bar{u} \gamma$ for all $\bar{u} \in \hat{\mathbf{K}}^{+}$. Set $\Psi^{\prime}(u)=\tilde{u}$. Then 


$$
\begin{aligned}
g(u, v) & =\operatorname{Tr}\left(\delta \mu\left(\tilde{u}^{\varphi} \bar{v}-\bar{u} \bar{v}^{\varphi}\right)\right) \\
& =\operatorname{Tr}\left(\delta \mu\left(\left[\tilde{u} \gamma^{-1}\right]^{\varphi} \tilde{v} \gamma^{-1}-\tilde{u} \gamma^{-1}\left[\tilde{v} \gamma^{-1}\right]^{\varphi}\right)\right) \\
& =\operatorname{Tr}\left(\delta \gamma^{-1} \gamma^{-\varphi} \mu\left(\tilde{u} \varphi \tilde{v}-\tilde{u} \tilde{v}^{\varphi}\right)\right) \\
& =\operatorname{Tr}\left(\mu\left(\tilde{u}^{\varphi} \widetilde{v}-\tilde{u} \widetilde{v}^{\varphi}\right)\right) .
\end{aligned}
$$

(3.8) If $\mu \in \hat{\mathbf{K}}^{\times}$and $\mu^{\varphi}=-\mu$ (if $r=2$ and $\mu=1$ ) then $\psi$ and $\Psi$ may be chosen so that $g(u, v)=\operatorname{Tr}\left(\mu\left(\bar{u}^{\varphi} \bar{v}-\overline{u v^{\varphi}}\right)\right)$.

Let $T^{*}\left(\hat{\mathbf{K}} / \mathbf{K}_{0}\right)$ be the subgroup of all elements of $T\left(\hat{\mathbf{K}} / \mathbf{K}_{0}\right)$ which fix the form of (3.8).

(3.9) Let $\hat{\mathbf{K}}_{0}$ be the subgroup of $\hat{\mathbf{K}}^{\times}$of order $r^{m}+1$. Let $\sigma$ be in the Galois group of $\hat{\mathbf{K}} / \mathrm{K}_{0}$. Set $T^{*}=T^{*}\left(\hat{\mathbf{K}} / \mathrm{K}_{0}\right)$. Then

(a) $\sigma \nu \in T^{*}$ for $\nu \in \hat{\mathbf{K}}^{x}$ if and only if $\mu^{\sigma} \mu^{-1} \nu^{\varphi} \nu=1$;

(b) $T^{*} \cap \hat{\mathbf{K}}^{\times}=\hat{K}_{0}$; and

(c) $T^{*} \hat{\mathbf{K}}^{\times}=T\left(\hat{\mathbf{K}} / \mathbf{K}_{0}\right)$.

Let $\nu \in \hat{\mathbf{K}}^{\times}$. Then $\sigma \nu \in T^{*}$ if and only if

$$
\begin{aligned}
\operatorname{Tr}\left(\mu\left(\bar{u}^{\varphi} \bar{v}-\bar{u} \bar{v}^{\varphi}\right)\right) & =\operatorname{Tr}\left(\mu\left([\sigma \nu \cdot \bar{u}]^{\varphi}(\sigma \nu \cdot \bar{v})-(\sigma v \cdot \bar{u})[\sigma \nu \cdot \bar{v}]^{\varphi}\right)\right) \\
& =\operatorname{Tr}\left(\left[\mu^{\sigma} \mu^{-1} \nu^{\varphi} \nu\right] \mu\left(\bar{u}^{\varphi} \bar{v}-\bar{u} \bar{v}^{\varphi}\right)\right)
\end{aligned}
$$

for all $u, v \in V$. This happens if and only if $\mu^{\sigma} \mu^{-1} \nu^{\varphi} \nu=1$. So condition (a) holds.

If $\sigma=1$ then from (a) we have $\nu^{\varphi} \nu=1$ or $\nu^{r m+1}=1$. So $T^{*} \cap \hat{\mathbf{K}}^{\times}=$ $\hat{\mathbf{K}}_{\mathbf{0}}$.

Let $\sigma$ be arbitrary in the Galois group of $\hat{\mathbf{K}} / \mathrm{K}_{0}$. To prove (c) we must show that there is a $\nu \in \hat{\mathbf{K}}^{\times}$such that $\sigma \nu \in T^{*}$. By (a) we want $\mu^{\sigma} \mu^{-1} \nu^{\varphi} \nu=1$. Since $\mu^{\varphi}=-\mu, \varphi$ fixes $\mu^{\sigma} \mu^{-1}$. That is, $\mu^{\sigma} \mu^{-1} \in \widetilde{\mathbf{K}}^{\times}$. The norm map $\epsilon \rightarrow$ $\epsilon \epsilon^{\varphi}$ takes $\hat{\mathbf{K}}^{\times}$onto $\widetilde{\mathbf{K}}^{\times}$. Therefore, there is a $\nu \in \hat{\mathbf{K}}^{\times}$such that $\nu^{\varphi} \nu=$ $\mu^{-\sigma} \mu \in \widetilde{\mathbf{K}}^{\times}$. But then $\mu^{\sigma} \mu^{-1} \nu^{\varphi} \nu=1$. Therefore $\sigma \nu \in T^{*}$. This completes the proof of (3.9).

We now have the following theorem.

(3.10) Assume (3.1) and (3.5). Set $\hat{\mathbf{K}}=\mathrm{GF}\left(r^{2 m}\right), \widetilde{\mathbf{K}}=\mathrm{GF}\left(r^{m}\right)$, and $\varphi$ : $\epsilon \rightarrow \epsilon^{r m}$ for $\epsilon \in \hat{\mathbf{K}}$. Fix $\mu \in \hat{\mathbf{K}}^{\times}$so that $\mu^{\varphi}=-\mu$ (if $r=2$ let $\mu=1$ ). Set

$$
g^{*}(\bar{u}, \bar{v})=\operatorname{Tr}\left(\mu\left(\bar{u}^{\varphi} \bar{v}-\bar{u} \bar{v}^{\varphi}\right)\right)
$$

for all $\bar{u}, \bar{v} \in \hat{\mathbf{K}}^{+}$where $\operatorname{Tr}: \widetilde{\mathbf{K}} \rightarrow \mathbf{K}$ is the trace map. Let $\hat{K}_{0}$ be the subgroup of $\widetilde{\mathbf{K}}^{\times}$of order $r^{m}+1$. Let $T^{*}(\hat{\mathbf{K}} / \mathbf{K})$ be the subgroup of $T(\hat{\mathbf{K}} / \mathbf{K})$ fixing $g^{*}$. There are monomorphisms 
$\psi: H \rightarrow T^{*}(\hat{\mathbf{K}} / \mathbf{K})$ and $\Psi: V \rightarrow \hat{\mathbf{K}}^{+}$(an isomorphism)

such that

(a) $\psi(x) \Psi(v)=\Psi(x v)$ all $x \in H, v \in V$;

(b) if $M=\langle x\rangle$ and $\psi(x)=\omega$ then $\omega \in \hat{K}_{0}$ and $\mathbf{K}(\omega)=\hat{\mathbf{K}}$;

(c) $\psi(H) \cap \hat{\mathbf{K}}^{\times}=\psi(M)$; and

(d) $g(u, v)=g^{*}(\Psi(u), \Psi(v))$ for all $u, v \in V$.

REMARK. Instead of assuming that $V$ is a minimal module, it is sufficient to assume here that $V l_{M}$ is irreducible, where $M$ is as in (3.3).

What happens in the case where $H$ is nilpotent and $N=1$ ? Then $M=$ $H$ is cyclic or $[H: M]=2$ by (3.3). If $M=H$ is cyclic then, by (3.10), $\psi(H)$ is a subgroup of the group $B$ described in (2.1) where $\Psi(V)=\hat{\mathbf{K}}^{+}$and $g$ is given by $g^{*}$.

Assume that $[H: M]=2$. Then $\psi(H) \hat{\mathbf{K}}^{\times} / \hat{\mathbf{K}}^{\times} \cong H / M$ has order 2. Thus if $H=\langle x, M\rangle$ then $\psi(x)=\varphi \nu$ where $\nu \in \hat{\mathbf{K}}^{\times}$. Recall that we now have a nilpotent group $H$. So $r>2$. Let $M_{0} \leqslant M$ be the Hall $2^{\prime}$-subgroup of $H$. If $\xi \in \psi\left(M_{0}\right) \leqslant \hat{K}_{0}$ then $(\varphi v)^{-1} \xi(\varphi v)=\xi^{\varphi}=\xi^{m}=\xi^{-1}$ since $\xi^{r^{m}+1}=1$. But $\xi=\psi(y)$ for some $y \in M_{0}$ and $x$ centralizes $y$. The map $\psi$ is an isomorphism so that $\xi=1$ or $M_{0}=1$. We have now proven that $H$ is a 2-group. Further, allowing $\xi$ to be any element of $\psi(M)$ proves that $x$ inverts $M$.

By (3.9)(a) we have $\mu^{\varphi} \mu^{-1} \nu^{\varphi} \nu=-\nu^{\varphi} \nu=1$ since $\varphi \nu \in T^{*}$. But then $\nu^{\varphi} \nu=-1$ or $(\varphi \nu)^{2}=-1$. The element $x \in H \backslash M$ was arbitrary. We conclude that $H \backslash M$ is a set of elements or order 4. By (3.3)(c), since $N=1$, we conclude that $H$ is quaternion. Also 4||$M \mid$ so that $4 \mid r^{m}+1$.

Clearly now $\dot{\psi(H)}$ is a subgroup of $G$ in example (2.2). The group $B$ is a 2-Sylow subgroup of $G$ and $\psi(H)$ is a 2-group. Therefore, there is a $d \in G$ such that $d \psi(H) d^{-1} \leqslant B$. If $x \in H$ and $u \in V$ set $\psi^{\prime}(x)=d \psi(x) d^{-1}$ and $\Psi^{\prime}(u)=d \Psi(u)$. Now $d$ fixes $g^{*}$ so we may replace $\psi$ and $\Psi$ by $\psi^{\prime}$ and $\Psi^{\prime}$. Then $\psi^{\prime}(H) \leqslant B$ of (2.2).

(3.11) Assume (3.1) and (3.5). Suppose $H=A$ is nilpotent and $N=1$. Then

(1) $H$ is cyclic and $H, V, g$ may be identified with $B_{0}, V, g$ of (2.1) where $B_{0} \leqslant B$; or

(2) $H$ is quaternion and $H, V, g$ may be identified with $B_{0}, V, g$ of (2.2) where $B_{0} \leqslant B$.

REMARK. As in (3.10) we need only assume that $\left.V\right|_{M}$ is irreducible (rather than $V$ is a minimal module) where $M$ is as in (3.3).

This completes the classification in the case where $\left.V\right|_{M}$ is homogeneous. 
We must now consider the case where $\eta_{M}$ is not homogeneous. Accordingly we change (3.5) to the following hypothesis.

(3.12) Hypothesis. Assume (3.1). Suppose $V_{M}=V_{1}+V_{2}$ where the $V_{i}$ are homogeneous components.

In this case we may interpret the results of (3.4) as follows: $\left[H: H_{0}\right]=$ 2, $H_{0} \geqslant M \geqslant N, \mathrm{~K}^{*}=\widetilde{\mathrm{K}}=\mathrm{GF}\left(r^{m}\right)$, and $U=V_{1}$.

We shall first find a canonical representation of $g$. Let $e \in \mathbf{K}[M]$ be a primitive central idempotent such that $e V_{1} \neq(0)$. Since $\left.V_{1}\right|_{M}$ is homogeneous, $e$ is unique. The subspaces $V_{1}, V_{2}$ are complementary and totally isotropic; so we may choose $v_{1} \in V_{1}, v_{2} \in V_{2}$ so that $g\left(v_{1}, v_{2}\right) \neq 0$. Then $0 \neq$ $g\left(v_{1}, v_{2}\right)=g\left(e v_{1}, v_{2}\right)=g\left(v_{1}, e^{\alpha} v_{2}\right)$. So $e^{\alpha} V_{2} \neq(0)$. This means $e^{\alpha}$ is the unique primitive central idempotent in $\mathrm{K}[M]$ such that $e^{\alpha} V_{2} \neq(0)$. Since $V_{1}$ and $V_{2}$ are not isomorphic, $e$ and $e^{\alpha}$ are orthogonal. Therefore, $\mathbf{K}[M] e_{0}=\mathbf{K}[M] e+\mathbf{K}[M] e^{\alpha}$ where $e_{0}=e+e^{\alpha}$.

By (3.4)(b), $\left.V_{1}\right|_{M}$ is irreducible. This means that, for any nonzero $v \in$ $V_{1}, \mathrm{~K}[M] e v=\mathrm{K}[M] v=V_{1}$. Choose $u_{1} \in V_{1}$ so that $\Psi\left(u_{1}\right)=1 \in \widetilde{\mathrm{K}}$. Similarly, for a nonzero $u^{\prime} \in V_{2}$ we have $K[M] e^{\alpha} u^{\prime}=V_{2}$. We may extend $\psi \mathbf{K}$-linearly to $\mathbf{K}[M]$. Then $\psi$ is a $\mathbf{K}$-linear isomorphism of $\mathbf{K}[M] e$ onto $\widetilde{\mathbf{K}}$. So for each $v \in V_{1}$ there is a unique $b \in \mathbf{K}[M] e$ so that $v=b u_{1}$. Similarly, for each $v^{\prime} \in V_{2}$ there is a unique $b^{\prime} \in \mathbf{K}[M] e^{\alpha}$ so that $v^{\prime}=b^{\prime} u^{\prime}$. Observe that $b^{\prime}$ will depend upon the choice $u^{\prime}, b^{\prime}=b^{\prime}\left(u^{\prime}\right)$. Now $b+b^{\prime} \in \mathbf{K}[M] e_{0}$ is the unique element of $\mathbf{K}[M] e_{0}$ such that $v+v^{\prime}=$ $\left(b+b^{\prime}\right)\left(u_{1}+u^{\prime}\right)$. That is, there is, for any $v^{\prime \prime} \in V$, a unique $b^{\prime \prime} \in \mathrm{K}[M] e_{0}$ so that $v^{\prime \prime}=b^{\prime \prime}\left(u_{1}+u^{\prime}\right)$.

We now determine a particular choice for $u^{\prime} \in V_{2}$. Since $V_{1}, V_{2}$ are complementary totally isotropic subspaces of $V$, and since $\mathrm{K}[M] e u_{1}=V_{1}$, the mapping $d \rightarrow g\left(u^{\prime}, d u_{1}\right)$ is a K-linear mapping of $\mathrm{K}[M] e$ onto $\mathbf{K}$. Therefore $f(\beta)=g\left(u^{\prime}, \psi^{-1}(\beta) u_{1}\right)$ is a $\mathbf{K}$-linear mapping of $\widetilde{\mathbf{K}}$ onto $\mathbf{K}$ where $\psi^{-1}$ is the inverse to the isomorphism $\psi: \mathbf{K}[M] e \rightarrow \widetilde{\mathbf{K}}$. There is then a $\gamma \in \widetilde{\mathbf{K}}^{\times}$so that $f(\beta)=\operatorname{Tr}(\gamma \beta)$ for $\beta \in \widetilde{\mathbf{K}}$ where $\operatorname{Tr}: \widetilde{\mathbf{K}} \rightarrow \mathbf{K}$ is a trace map. Let $d=\psi^{-1}\left(\gamma^{-1}\right) \in \mathbf{K}[M]$ e. Set $u_{2}=d^{\alpha} u^{\prime} \in V_{2}$. Then, for $b \in \mathbf{K}[M] e$ and $\psi(b)=\beta$,

$$
\begin{aligned}
g\left(u_{2}, b u_{1}\right) & =g\left(d^{\alpha} u^{\prime}, b u_{1}\right)=g\left(u^{\prime}, d b u_{1}\right) \\
& =g\left(u^{\prime}, \psi^{-1}\left(\gamma^{-1} \beta\right) u_{1}\right)=f\left(\gamma^{-1} \beta\right) \\
& =\operatorname{Tr}\left(\gamma \gamma^{-1} \beta\right)=\operatorname{Tr}(\beta) \\
& =\operatorname{Tr}(\psi(b)) .
\end{aligned}
$$

So we replace $u^{\prime}$ by $u_{2}$. 
Let $u, v \in V$. Choose unique elements $b, c \in \mathbf{K}[M] e_{0}$ so that $u=$ $b\left(u_{1}+u_{2}\right)$ and $v=c\left(u_{1}+u_{2}\right)$. We now compute a representation for $g$.

$$
\begin{aligned}
g(u, v) & =g\left(b\left(u_{1}+u_{2}\right), c\left(u_{1}+u_{2}\right)\right) \\
& =g\left(b u_{1}, c u_{2}\right)+g\left(b u_{2}, c u_{1}\right) \\
& =g\left(b e^{\alpha} u_{2}, c e u_{1}\right)-g\left(c e^{\alpha} u_{2}, b e u_{1}\right) \\
& =g\left(u_{2},\left[\left(b^{\alpha} e\right)(c e)-\left(c^{\alpha} e\right)(b e)\right] u_{1}\right) \\
& =\operatorname{Tr}\left(\psi\left(b^{\alpha} e\right) \psi(c e)-\psi\left(c^{\alpha} e\right) \psi(b e)\right) .
\end{aligned}
$$

(3.13) Let $e$ be a primitive central idempotent of $\mathbf{K}[M]$ such that $e V_{1} \neq(0)$. Set $e_{0}=e+e^{\alpha}$. The map $\psi$ extends $\mathrm{K}$-linearly to $\mathrm{K}[M]$. Then $\psi: \mathrm{K}[M] e \rightarrow \widetilde{\mathbf{K}}$ is a $\mathrm{K}$-isomorphism Let $u_{1} \in V_{1}$ be chosen so that $\Psi\left(u_{1}\right)=1$. There is a nonzero $u_{2} \in V_{2}$ so that

(1) for each $v \in V$ there is a unique $b \in K[M] e_{0}$ so that $v=$ $b\left(u_{1}+u_{2}\right)$

(2) if $b, c \in \mathbf{K}[M] e_{0}$ then

$$
g\left(b\left(u_{1}+u_{2}\right), c\left(u_{1}+u_{2}\right)\right)=\operatorname{Tr}\left(\psi\left(b^{\alpha} e\right) \psi(c e)-\psi(b e) \psi\left(c^{\alpha} e\right)\right)
$$

where $\operatorname{Tr}: \widetilde{\mathbf{K}} \rightarrow \mathbf{K}$ is the trace map.

We may now build maps $\psi^{\prime}$ and $\Psi^{\prime}$ for $M$ and $V_{2}$ analogous with $\psi$ and $\Psi$ for $M$ and $V_{1}$.

(3.14) For $x \in M$ set $\psi^{\prime}(x)=\psi \alpha(x)=\psi\left(x^{-1}\right)$. Then $\psi^{\prime}: M \rightarrow \widetilde{\mathbf{K}}^{\times}$is a monomorphism. We may extend $\psi^{\prime} \mathrm{K}$-linearly to $\mathrm{K}[M]$. Then $\psi^{\prime}$ : $\mathbf{K}[M] e^{\alpha} \rightarrow \widetilde{\mathbf{K}}$ is a $\mathbf{K}$-isomorphism. If $u \in V_{2}$ there is a unique $b \in \mathbf{K}[M] e^{\alpha}$ so that $b u_{2}=u$. Set $\Psi^{\prime}(u)=\psi^{\prime}(b)$. Then $\Psi^{\prime}$ is a $\mathrm{K}$-linear isomorphism of $V_{2}$ onto $\widetilde{\mathrm{K}}^{+}$such that $\psi^{\prime}(x) \Psi^{\prime}(u)=\Psi^{\prime}(x u)$ for any $x \in M$ and $u \in V_{2}$.

Most of this is straightforward from the preceding arguments. Observe that

$$
\begin{aligned}
\psi^{\prime}(x) \Psi^{\prime}(u) & =\psi \alpha(x) \psi^{\prime}(b)=\psi\left(x^{-1}\right) \psi\left(b^{\alpha}\right) \\
& =\left(x^{-1} b^{\alpha}\right)=\psi\left([b x]^{\alpha}\right)=\Psi^{\prime}\left(b x u_{2}\right) \\
& =\Psi^{\prime}(x u) .
\end{aligned}
$$

We are now in a position to give a canonical form for $M, V, g$. Let $\left\{e_{1}, e_{2}\right\}$ be a $\widetilde{\mathrm{K}}$-basis for a 2 -dimensional $\widetilde{\mathrm{K}}$-space $W$. Suppose $u, v \in V$ and $b, c \in \mathbf{K}[M] e_{0}$ so that $u=b\left(u_{1}+u_{2}\right)$ and $v=c\left(u_{1}+u_{2}\right)$. Set $\Psi_{0}(u)=$ $\psi^{\prime}(b e) e_{1}+\psi^{\prime}\left(b e^{\alpha}\right) e_{2}$. Let

$$
\bar{g}\left(\psi(b e) e_{1}+\psi^{\prime}\left(b e^{\alpha}\right) e_{2}, \psi(c e) e_{1}+\psi^{\prime}\left(c e^{\alpha}\right) e_{2}\right)=g(u, v) .
$$

If $x \in M$ we set 


$$
\psi_{0}(x)=\left[\begin{array}{ll}
\psi(x) & \\
& \psi^{\prime}(x)
\end{array}\right]
$$

Then $\psi_{0}(x)$ is a linear transformation of $W$ given in the basis $\left\{e_{1}, e_{2}\right\}$.

(3.15) If $x \in M$ and $u \in V$ then $\psi_{0}, \Psi_{0}$ are monomorphisms such that $\psi_{0}(x) \Psi_{0}(u)=\Psi_{0}(x u)$. Further, if $v \in V$ also, then $\bar{g}\left(\Psi_{0}(u), \Psi_{0}(v)\right)=$ $g(u, v)$. If $\Psi_{0}(u)=\beta_{1} e_{1}+\beta_{2} e_{2}$ and $\Psi_{0}(v)=\gamma_{1} e_{1}+\gamma_{2} e_{2}$ then $\bar{g}\left(\beta_{1} e_{1}+\beta_{2} e_{2}, \gamma_{1} e_{1}+\gamma_{2} e_{2}\right)=\operatorname{Tr}\left(\beta_{2} \gamma_{1}-\beta_{1} \gamma_{2}\right)$ where $\operatorname{Tr}: \widetilde{\mathbf{K}} \rightarrow \mathrm{K}$ is the trace map.

This follows directly from (3.13) and (3.14).

The object is to extend $\psi_{0}$ to all of $H$. The extension will follow since the existence of $\psi_{0}$ upon $M$ forces the uniqueness of any extension. We prove this below.

$$
\left.T^{\dagger}(\tilde{\mathbf{K}} / \mathbf{K})=\left\langle\left[\begin{array}{r}
1 \\
-1
\end{array}\right],\left[\begin{array}{ll}
\sigma \nu & \\
& \sigma \nu^{-1}
\end{array}\right]\right| \sigma \in \text { Aut }(\widetilde{\mathbf{K}}), \nu \in \widetilde{\mathbf{K}}^{x}\right\rangle .
$$

Then $T^{\dagger}(\widetilde{\mathbf{K}} / \mathrm{K})$ is a group of $\mathbf{K}$-linear transformations of $V$ written in the $\widetilde{\mathbf{K}}$-basis $\left\{e_{1}, e_{2}\right\}$. Further, $\tau^{\dagger}(\widetilde{\mathrm{K}} / \mathrm{K})$ is the group of all $\mathbf{K}$-linear transformations of $V$ which normalize $\psi_{0}(M)$ and fix the form $\bar{g}$ given in (3.15).

Let $\tau=\left[{ }_{-1}^{1}\right]$. Let $u=\beta_{1} e_{1}+\beta_{2} e_{2}$ and $v=\gamma_{1} e_{1}+\gamma_{2} e_{2}$. Then

$$
\begin{aligned}
\bar{g}(\tau u, \tau v) & =\operatorname{Tr}\left(\left(\beta_{1}\right)\left(-\gamma_{2}\right)-\left(-\beta_{2}\right)\left(\gamma_{1}\right)\right) \\
& =\operatorname{Tr}\left(\beta_{2} \gamma_{1}-\beta_{1} \gamma_{2}\right)=\bar{g}(u, v) .
\end{aligned}
$$

Let

$$
\tau_{0}=\left[\begin{array}{cc}
\sigma \nu & \\
& \sigma \nu^{-1}
\end{array}\right] \text { for } \sigma \in \operatorname{Aut}(\widetilde{\mathbf{K}}), \nu \in \widetilde{\mathbf{K}}^{\times}
$$

Then

$$
\begin{aligned}
\bar{g}\left(\tau_{0} u, \tau_{0} v\right) & =\operatorname{Tr}\left(\left(\sigma \nu^{-1} \cdot \beta_{2}\right)\left(\sigma \nu \cdot \gamma_{1}\right)-\left(\sigma \nu \cdot \beta_{1}\right)\left(\sigma \nu^{-1} \cdot \gamma_{2}\right)\right) \\
& =\operatorname{Tr}\left(\left[\beta_{2} \gamma_{1}-\beta_{1} \gamma_{2}\right]^{\sigma^{-1}}\right) \\
& =\operatorname{Tr}\left(\beta_{2} \gamma_{1}-\beta_{1} \gamma_{2}\right) \\
& =\bar{g}(u, v) .
\end{aligned}
$$

Therefore $T^{\dagger}(\tilde{\mathbf{K}} / \mathbf{K})=T^{\dagger}$ fixes $\overline{\boldsymbol{g}}$. Observe that 


$$
T_{0}^{\dagger}=\left\langle\left[\begin{array}{cc}
\sigma \nu & \\
& \sigma \nu^{-1}
\end{array}\right] \mid \sigma \in \operatorname{Aut}(\widetilde{\mathbf{K}}), \nu \in \widetilde{\mathbf{K}}^{\times}\right\rangle
$$

is the stabilizer in $T^{\dagger}$ of $\widetilde{\mathbf{K}} e_{1}$. Since

$$
\psi_{0}(M)=\left\{\left[\begin{array}{ll}
\xi & \\
& \xi^{-1}
\end{array}\right] \mid \xi=\psi(x), x \in M\right\}
$$

we conclude that $T^{\dagger}$ normalizes $\psi_{0}(M)$.

Let $G$ be the group of all K-linear transformations of $V$ which fix $\vec{g}$ and normalize $\psi_{0}(M)$. Let $G_{0}$ be the stabilizer in $G$ of $\widetilde{K}_{e_{1}}$. Then $G \geqslant$ $T^{\dagger}$ and $G_{0} \geqslant T_{0}^{\dagger}$. Since $G$ normalizes $\psi_{0}(H)$ and $\widetilde{K}_{e_{1}}, \widetilde{\mathbf{K}} e_{2}$ are nonisomorphic $\psi_{0}(H)$-modules $\left(\left.V_{1}\right|_{M} \neq\left. V_{2}\right|_{M}\right)$, we conclude that $\left[G: G_{0}\right]=2$. Since $\left[T^{\dagger}: T_{0}^{\dagger}\right]=2$, if $G_{0}=T_{0}^{\dagger}$ then $G=T^{\dagger}$. To complete the proof we need only show that $G_{0}=T_{0}^{\dagger}$.

Since $G_{0}$ fixes the form $\bar{g}$, the action of $G_{0}$ upon $\widetilde{\mathbf{K}} e_{1}$ is contragredient to the action of $G_{0}$ upon $\widetilde{K}_{e_{2}}$. Therefore $G_{0}$ is faithful upon $\widetilde{K}_{e_{1}}$.

Now let $G_{0}^{*}$ be the group induced by $G_{0}$ upon $\widetilde{\mathbf{K}} e_{1}$. The group induced by $\psi_{0}(M)$ upon $\widetilde{\mathbf{K}} e_{1}$ is $\psi(M)$. So $\psi(M)$ is a normal subgroup of $G_{0}^{*}$. But $\left.V_{1}\right|_{M}$ is irreducible by (3.4) (b) so that $\psi_{0}(M)$ is irreducible upon $\widetilde{K}_{e_{1}}$. By (1.2), then, $G_{0}^{*}$ is a subgroup of $T(\widetilde{K} / \mathbf{K})$. That is, $\left|G_{0}^{*}\right| \leqslant|T(\widetilde{K} / \mathbf{K})|=$ $\left|T_{0}^{\dagger}\right|$. Since $\left|G_{0}^{*}\right|=\left|G_{0}\right|$ and $G_{0} \geqslant T_{0}^{\dagger}$ we must have $G_{0}=T_{0}^{\dagger}$. So $G=$ $\mathrm{T}^{\dagger}$ and the proof is complete.

For $x \in H$, the equation $\psi_{0}^{\prime}(x) \Psi_{0}(u)=\Psi_{0}(x u)$ for $u \in V$ defines a K-linear transformation of $W$ which must normalize $\psi_{0}(M)$. Also $\psi_{0}^{\prime}(x)=$ $\psi_{0}(x)$ if $x \in M$. By (3.13) and (3.15), $\psi_{0}^{\prime}(x)$ will fix $\bar{g}$. It is easy to verify that $\psi_{0}^{\prime}$ is a homomorphism. Since $\psi_{0}^{\prime}$ is a monomorphism upon $M, \psi_{0}^{\prime}$ is a monomorphism upon $H$. By (3.16), $\psi_{0}^{\prime}(x) \in T^{\dagger}$. We have proved the following theorem.

(3.17) Assume (3.1) and (3.12). Set $\widetilde{\mathbf{K}}=\mathrm{GF}\left(r^{m}\right)$. Let $T^{\dagger}(\widetilde{\mathbf{K}} / \mathrm{K}), W, \bar{g}$, and $\left\{e_{1}, e_{2}\right\}$ be as in (3.16). There are monomorphisms $\psi_{0}: H \rightarrow T^{\dagger}(\widetilde{\mathrm{K}} / \mathrm{K})$ and $\Psi_{0}: V \rightarrow W(a$ K-isomorphism) such that

(a) $\psi_{0}(x) \Psi_{0}(u)=\Psi_{0}(x u)$ all $x \in H, u \in V$;

(b) if $M=\langle x\rangle$ then $\psi_{0}(x)=\left[\begin{array}{ll}\omega & \\ & \omega^{-1}\end{array}\right]$ where $\omega \in \widetilde{K}^{\times}$and $\mathbf{K}(\omega)=\widetilde{\mathbf{K}}$;

(c) $\psi_{0}(H) \cap\left\langle\left[\begin{array}{ll}\xi & \\ & \xi^{-1}\end{array}\right] \mid \xi \in \widetilde{\mathbf{K}}^{x}\right\rangle=\psi_{0}(M)$; and 
(d) $g(u, v)=\bar{g}\left(\Psi_{0}(u), \Psi_{0}(v)\right)$ all $u, v \in V$.

Remark. We need not assume that $V$ is a minimal module; $\left.V_{1}\right|_{M}$ is irreducible when $M$ as in (3.3) is sufficient.

Again we turn to the case where $H=A$ is nilpotent and $N=1$. Then $M=H$ or $[H: M]=2$. In the former case $V$ is irreducible as in $\mathrm{K}[M]$ module. By hypothesis (3.12), $\left.V\right|_{M}$ is reducible. We conclude that $[H: M]=$ 2 and $r>2$.

We may write $M=\left\langle x_{1}\right\rangle \times\left\langle x_{2}\right\rangle$ where $x_{1}$ has $2^{\prime}$-order and $x_{2}$ has 2 power order. By (3.17)

$$
\psi_{0}\left(x_{1}\right)=\bar{x}_{1}=\left[\begin{array}{ll}
\omega & \\
& \omega^{-1}
\end{array}\right], \quad \psi_{0}\left(x_{2}\right)=\bar{x}_{2}=\left[\begin{array}{ll}
\eta & \\
& \eta^{-1}
\end{array}\right]
$$

and, for $y \in H \backslash M$ of 2-power order,

$$
\psi_{0}(y)=\bar{y}=\left[\begin{array}{r}
\sigma \nu^{-1} \\
-\sigma \nu
\end{array}\right]
$$

for some $\sigma \in$ Aut $(\widetilde{\mathbf{K}})$ and $\nu \in \widetilde{\mathbf{K}}^{\times}$.

Since $y^{2} \in M$, we have

$$
\bar{y}^{2}=\left[\begin{array}{cc}
-\sigma^{2} \nu^{-\sigma} \nu & \\
& -\sigma^{2} \nu^{\sigma} \nu^{-1}
\end{array}\right]=\left[\begin{array}{ll}
\xi & \\
& \xi^{-1}
\end{array}\right]
$$

for some $\xi \in \widetilde{K}^{\times}$. We must have $\sigma^{2}=1$ and $-\nu^{-\sigma} \nu=\xi$. There are two possibilities.

$$
\sigma=1 \text { or } m=2 n \text { and } \sigma: \epsilon \rightarrow \epsilon^{r^{n}} \text {. }
$$

First consider the case where $\sigma=1$. This will give rise to example (2.3). Now $\bar{y}$ inverts $\left\langle\bar{x}_{1} \bar{x}_{2}\right\rangle=\psi_{0}(M)$ by conjugation. If we choose $\bar{y}$ of 2-power order then $\left.\bar{x}_{1}, \bar{y}\right\rangle$ must be a nilpotent group. Since $\bar{y}$ inverts $\bar{x}_{1}$ and $\bar{x}_{1}$ has odd order, $\bar{x}_{1}=1$. The map $\psi_{0}$ is an isomorphism upon $H$. Therefore $H$ is a 2-group. In this case $\sigma=1$ so that $-\nu^{-\sigma} \nu=-\nu^{-1} \nu=-1$. So $\bar{y}$ has order 4. Now $y \in H \backslash M$ was arbitrary so that all $y \in H \backslash M$ have order 4. But $y$ inverts $M$ by conjugation. So $H$ is quaternion. Since 4||$M \mid$ and $|M| \mid r^{m}-1$ we have $r^{m} \equiv 1(\bmod 4)$.

Write $\nu=\xi \theta$ where $\zeta$ has $2^{\prime}$-order and $\theta$ has 2-power order in $\widetilde{\mathbf{K}}^{x}$. Let 


$$
d=\left[\begin{array}{cc}
\epsilon & \\
& \epsilon^{-1}
\end{array}\right] \text { where } \epsilon \in \widetilde{\mathbf{K}}^{\times} \text {and } \epsilon^{2}=\zeta
$$

Set

$$
\Psi_{0}^{\prime}(u)=d \Psi_{0}(u) \quad \text { for } u \in V
$$

and

$$
\psi_{0}^{\prime}(z)=d \psi_{0}(z) d^{-1} \quad \text { for } z \in H .
$$

Since $d$ fixes $\bar{g}$, we may replace $\psi_{0}, \Psi_{0}$ by $\psi_{0}^{\prime}, \Psi_{0}^{\prime}$. But $d$ centralizes $\psi_{0}(M)$. So $\psi_{0}=\psi_{0}^{\prime}$ upon $M$. Further,

$$
\psi_{0}^{\prime}(y)=\left[\begin{array}{cc}
\theta^{-1} \\
-\theta
\end{array}\right] .
$$

Therefore $\psi_{0}^{\prime}(H) \leqslant B$ of Example (2.3).

Second, we consider the case where $\sigma: \epsilon \rightarrow \epsilon^{r n}$ where $m=2 n$. Then $-\nu^{-\sigma} \nu=-\nu^{1-r^{n}}=\xi$.

Conjugating,

$$
\bar{x}_{1} \bar{y}=\left[\begin{array}{ll}
\omega^{-\sigma} & \\
& \omega^{\sigma}
\end{array}\right]=\left[\begin{array}{ll}
\omega & \\
& \omega^{-1}
\end{array}\right]
$$

since $H$ is nilpotent. Then $\omega^{-\sigma}=\omega^{-r n}=\omega$ so that $\left.K \omega\right\rangle \mid$ divides $r^{n}+1$. The Hall 2'-subgroup of $H$ is now a subgroup of $D$ in example (2.4).

Conjugating again,

$$
\bar{x}_{2}^{\bar{y}}=\left[\begin{array}{ll}
\eta^{-\sigma} & \\
& \eta^{\sigma}
\end{array}\right] \text {. }
$$

By (3.3) the 2-Sylow subgroup of $H$ must be quaternion, dihedral, or semidihedral. That is $\eta^{-\sigma}=\eta^{2 t-1}$ where $|\eta \eta\rangle=2^{t}$ or $2^{t+1}$. Suppose $\eta$ has order $2^{t}$. Then $\eta^{r^{n}}=\eta$ or $\eta \in \mathrm{GF}\left(r^{n}\right)$. So $4 \mid r^{n}-1$ since $|\eta \eta\rangle=2^{t} \geqslant 4$.

Suppose $\eta$ has order $2^{t+1}$ (where here $t \geqslant 2$ since the 2-Sylow subgroup is semidihedral). Therefore $\eta^{r^{n}}=\eta^{2^{t}-1}=-\eta^{-1}$ or $\eta^{r_{-1}}=-1$. But then $\eta^{2}$ has order divisible by 4 and lies in $\operatorname{GF}\left(r^{n}\right)$. So again $4 \mid r^{n}-1$.

Let $\rho \in \widetilde{K}^{\times}$have order $2^{s+1}$ where $2^{s} \mid v^{n}-1$. Let

$$
\bar{x}_{0}=\left[\begin{array}{ll}
\rho & \\
& \rho^{-1}
\end{array}\right] \text {. }
$$


Then $H_{1}=\left\langle\psi_{0}(H), \bar{x}_{0}\right\rangle \geqslant \psi_{0}(H)$ and is nilpotent. Since $2^{s+1} \mid r^{m}-1, \rho$ is a generator for the 2-Sylow subgroup of $\widetilde{\mathbf{K}}^{\times}$.

Write $\nu=\zeta \theta$ where $\zeta$ has $2^{\prime}$-order and $\theta$ has 2-power order in $\widetilde{\mathbf{K}}^{\times}$. Replace $\bar{y}$ by $\bar{y}^{\prime}=\bar{y} \bar{w}$,

$$
\bar{w}=\left[\begin{array}{r}
\theta^{-1} \\
\theta
\end{array}\right] \in H_{1} .
$$

We now have $\nu$ a $2^{\prime}$-element.

The group $\left\langle\bar{y}, \bar{x}_{0}\right\rangle$ is semidihedral so that $\bar{y}$ will have order 2 or 4 . We show that it has order 4. Suppose it has order 2. Then $\vec{y}^{2}=1$ so that $-\nu^{-\sigma} \nu=\xi=1$ or $\nu^{\sigma}=\nu^{n}=-\nu$.

But both $\nu$ and $\nu^{\sigma}$ have odd order so that this situation is impossible. Therefore, $\bar{y}$ has order 4 and so $-\nu^{-\sigma} \nu=\xi=-1$ or $\nu^{\sigma}=\nu=\nu^{r n}$. In this case $\nu \in \mathrm{GF}\left(r^{n}\right)$.

We may now choose $\lambda \in \mathrm{GF}\left(r^{n}\right)$ so that $\lambda^{2}=\nu$. Let

$$
d=\left[\begin{array}{ll}
\lambda & \\
& \lambda^{-1}
\end{array}\right] .
$$

Let $\psi_{0}^{\prime}(x)=d \psi_{0}(x) d^{-1}, \Psi_{0}^{\prime}(u)=d \Psi_{0}(u)$, and $H_{1}^{\prime}=d H_{1} d^{-1}$. We may replace $\psi_{0}, \Psi_{0}$ by $\psi_{0}^{\prime}, \Psi_{0}^{\prime}$ since $d$ fixes $\bar{g}$. Then $\bar{y}$ becomes $\bar{y}=\left[{ }_{-\sigma}{ }^{\sigma}\right]$. Now $\psi_{0}^{\prime}(H) \leqslant H_{1}^{\prime} \leqslant B$ of example (2.4).

Summing up, we have:

(3.19) Assume (3.1) and (3.12). Suppose $H=A$ is nilpotent and $N=1$. Then $r>2$ and

(1) $H$ is quaternion and $H, V, g$ may be identified with $B_{0}, V, g$ of (2.3) where $B_{0} \leqslant B$; or

(2) $H$ has a quaternion, dihedral, or semihedral 2-Sylow subgroup and $H, V, g$ may be identified with $B_{0}, V, g$ of (2.4) where $B_{0} \leqslant B$.

REMARK . The remark following (3.17) applies here.

Combining both (3.11) and (3.19) we obtain the following theorem.

(3.20) Assume (3.1). Suppose $H=A$ is nilpotent and $N=1$. Then $H, V, g$ may be identified with $B_{0}, V, g$ of one of the examples (2.1)-(2.4) where $B_{0} \leqslant B$.

We can state certain other restraints upon the choices (2.1)-(2.4) when $H$ is nilpotent. 
(3.21) (1) In example (2.2), $B$ is reducible upon $V$ unless $m=1$.

(2) In example (2.3), $B$ is reducible upon $V$ unless $m=2^{s}$ is a power of 2.

Consider (1) first. Then $B=\langle\varphi \nu, Q\rangle$, and $\nu^{\varphi}=-\nu^{-1}$. Suppose $Q=\langle\omega\rangle$ has order $2^{t}$. Since $2^{t} \mid r^{m}+1$ we have $r^{m} \equiv-1(\bmod 4)$. Now $m$ must be odd. We may factor $r^{m}+1$ as

$$
r^{m}+1=(r+1)\left(r^{m}-r^{m-1}+\cdots+r^{2}-r+1\right) .
$$

The second factor is odd so that $2^{t} \mid r+1$. Therefore $\omega \in \mathrm{GF}\left(r^{2}\right)$. By (3.10) (b), $\mathbf{K}(\omega)=\hat{\mathbf{K}}=\mathrm{GF}\left(r^{2 m}\right)$. So $m=1$.

Consider (2) next. Then $B=\langle\tau, Q\rangle$ where $Q_{0}=\langle\omega\rangle$ has order $2^{t}$. Since $m=2^{s} n$ for odd $n$ we may factor $r^{m}-1$ as

$$
r^{m}-1=\left(r^{2 s}-1\right)\left(r^{2^{s}(n-1)}+\cdots+r^{2^{s}}+1\right) \text {. }
$$

Again the second factor is odd so that $2^{t} \mid r^{2^{s}}-1$. But then $\omega \in \operatorname{GF}\left(r^{2 s}\right)$. By (3.17)(b) we have $\mathrm{K}(\omega)=\widetilde{\mathrm{K}}=\mathrm{GF}\left(r^{m}\right)$. So $n=1$ and $m$ is a power of 2.

(3.22) Let $S$ be as in (2.4). Suppose

$$
y=\left[\begin{array}{ll}
\nu & \\
& \nu^{-1}
\end{array}\right]
$$

generates $Q$. Let $\mathrm{K}^{*}=\mathrm{GF}\left(r^{n}\right)$ where $m=2 n$. Let $T$ be a maximal subgroup of $S$.

(1) $T=Q$ is cyclic and $V l_{T}=\widetilde{\mathbf{K}} e_{1}+\widetilde{\mathbf{K}} e_{2}$ is the sum of two totally isotropic subspaces.

(2) $T=\left\langle\tau y, y^{2}\right\rangle$ is dihedral and $\left.V\right|_{T}=\left(K^{*} e_{1}+K^{*} \nu e_{2}\right) \dot{+}\left(K^{*} \nu e_{1}+K^{*} e_{2}\right)$ is the sum of two totally isotropic subspaces.

(3) $T=\left\langle\tau, y^{2}\right\rangle$ is quaternion and $V_{T}=\left(\mathrm{K}^{*}(1+\nu) e_{1}+\mathrm{K}^{*}(1-v) e_{2}\right) \dot{+}$ $\left(\mathrm{K}^{*}\left(1+\nu^{-1}\right) e_{1}+\mathrm{K}^{*}\left(1-\nu^{-1}\right) e_{2}\right)$ is the sum of two nonsingular mutually orthogonal subspaces.

Further, $y\left(\mathbf{K}^{*}(1+\nu) e_{1}+\mathbf{K}^{*}(1-\nu) e_{2}\right)=\left(\mathbf{K}^{*}\left(1+\nu^{-1}\right) e_{1}+\mathbf{K}^{*}\left(1-\nu^{-1}\right) e_{2}\right)$.

Part (1) is obvious. Now $\nu^{2} \in K^{*}$ so that all subspaces are invariant under $y^{2}$. In (2) we have $\tau y e_{1}=-\nu e_{2}$ and $\tau y \nu e_{2}=e_{1}$. So the decomposition in (2) is $T$-invariant. Further, $y\left(K^{*} e_{1}+K^{*} v e_{2}\right)=K^{*} v e_{1}+K^{*} e_{2}$. If $\alpha_{1}, \alpha_{2}, \beta_{1}, \beta_{2} \in K^{*}$ then 


$$
\begin{aligned}
& g\left(\alpha_{1} e_{1}+\alpha_{2} \nu e_{2}, \beta_{1} e_{1}+\beta_{2} \nu e_{2}\right)=\operatorname{Tr}\left(\alpha_{2} \nu \beta_{2}-\beta_{2} \nu \alpha_{1}\right) \\
& =\operatorname{tr}\left(\alpha_{2} \nu \beta_{2}+\alpha_{2}{ }^{r^{n}} \nu^{{ }^{r n}} \beta_{2}{ }^{r^{n}}-\beta_{2} \nu \alpha_{1}-\beta_{2}{ }^{{ }^{n}} \nu^{r^{n}} \alpha_{1}{ }^{{ }^{n}}\right) \\
& =\operatorname{tr}(0)
\end{aligned}
$$

where $\operatorname{tr}: \mathbf{K}^{*} \rightarrow \mathbf{K}$ is the trace and $\boldsymbol{\gamma}+\boldsymbol{\gamma}^{\boldsymbol{r}^{n}}$ is the trace from $\widetilde{\mathbf{K}} \rightarrow \mathbf{K}^{*}$. Here we have used the fact that $\nu^{r^{n}}=-\nu$. So the two subspaces (being conjugated by $y$ ) are totally isotropic.

The last part of (3) is obvious since $\nu^{2} \in \mathrm{K}^{*}$. Since $V$ is nonsingular, we need only prove that the two subspaces are mutually orthogonal.

Let $\alpha_{1}, \alpha_{2}, \beta_{1}, \beta_{2} \in K^{*}$. Then

$$
\begin{aligned}
g\left(\alpha_{1}(1\right. & \left.+\nu) e_{1}+\alpha_{2}(1-\nu) e_{2}, \beta_{1}\left(1+\nu^{-1}\right) e_{1}+\beta_{2}\left(1-\nu^{-1}\right) e_{2}\right) \\
& =\operatorname{Tr}\left(\alpha_{2} \beta_{1}\left(\nu^{-1}-\nu\right)-\alpha_{1} \beta_{2}\left(\nu-\nu^{-1}\right)\right) \\
& =\operatorname{tr}\left(\left(\alpha_{2} \beta_{1}+\alpha_{1} \beta_{2}\right)\left[\left(\nu^{-1}-\nu\right)+\left(\nu^{-1}-\nu\right)^{r^{n}}\right]\right) \\
& =\operatorname{tr}(0)=0
\end{aligned}
$$

where $\operatorname{tr}: K^{*} \rightarrow \mathbf{K}$ is the trace. Here we have used the fact that $\nu^{\boldsymbol{r}^{n}}=-\boldsymbol{\nu}$.

The $\tau$-invariance of the subspaces is easy to show. So (3) holds.

4. Some symplectic primitive $K[G]$-modules. Suppose $H$ is a group; $\mathbf{k}$ is a field; $U$ is an irreducible $\mathbf{k}[H]$-module; and $g$ is a nonsingular symplectic form upon $U$ fixed by $H$. We say that $W$ symplectically induces $U$ if

(1) $W$ is a $\mathrm{k}\left[H_{0}\right]$-submodule of $U$ for a subgroup $H_{0}$ of $H$;

(2) $g$ is nonsingular upon $W$;

(3) $\left.W\right|^{H} \cong U$; and

(4) if $x, y \in H$ where $x H_{0} \neq y H_{0}$ then $x W$ and $y W$ are orthogonal. We say that $U$ is symplectic primitive if $U$ is not symplectically induced by any proper subspace $W$ of $U$.

From [4, (3.7)] we discover that:

(4.1) If $U$ is symplectic primitive then $U$ is a minimal $\mathrm{K}[H]$-module.

In this section we shall analyze nilpotent groups $H$ which are symplectic primitive. We fix the following assumption for this section.

(4.2) HYPOTHESIS. $\mathrm{K}=\mathrm{GF}(r)$ for a prime $r>2$.

(1) $V$ is a $2 m$-dimensional $\mathrm{K}$-vector space.

(2) $g: V \times V \rightarrow K$ is a nonsingular symplectic form upon $V$.

(3) $H$ is a nilpotent group.

(4) $V$ is a faithful symplectic primitive $\mathrm{K}[H]$-module where $H$ fixes the form $g$. 
By (3.1) and (3.20) we may view $H, V, g$ as $B_{0}, V, g$ where $B_{0} \leqslant B$ for one of the examples (2.1)-(2.4). We now assume that $H \leqslant B$ and that we are considering one of the examples (2.1)-(2.4).

(4.3) If $H$ is described by (2.2) then $m=1$.

This follows immediately from (3.21)(1).

(4.4) If $B_{0} \leqslant B$ is any quaternion subgroup of (2.2) or (2.3) for $m=1$ then $V$ is a symplectic primitive $B_{0}$-module.

First observe that, since $B_{0}$ is quaternion and $\operatorname{dim} V=2, V$ must be irreducible. But since $\operatorname{dim} V=2$, it has no proper nonsingular $\mathrm{K}$-subspaces. Therefore $V$ must be symplectic primitive.

(4.5) If $H$ is described by (2.3) then $m=1$ and $r \equiv 1(\bmod 4)$.

Suppose $V$ is symplectically induced as a $B$-module for a $B_{0}$-submodule $W$ of $V$ where $\left[B: B_{0}\right]=2$. Then $V=W+z W$ where $z \in B \backslash B_{0}$. If $H \leqslant B_{0}$ then $W$ and $z W$ are proper $H$-modules so $V_{H}$ is reducible. By (4.2) we must then have $B_{0} H=B$. So $z$ may be chosen from $H$. But then, as a $H$-module, $V$ is symplectically induced by the $H_{0}=H \cap B_{0}$-module $W$. The symplectic primitivity of $V l_{H}$ implies the symplectic primitivity of $\left.V\right|_{B}$.

Recall that $B=\langle\tau, Q\rangle$ where $Q=\langle y\rangle$ and

$$
y=\left[\begin{array}{ll}
\nu & \\
& \nu^{-1}
\end{array}\right]
$$

for $\nu \in \widetilde{\mathbf{K}}=\mathrm{GF}\left(r^{m}\right)$ of order $2^{t} \mid \boldsymbol{r}^{m}-1$. By (3.21)(2), $m=2^{s}$ is a power of 2.

If $r \equiv-1(\bmod 4)$ assume that $s>1$. In any case, assume that $s>0$. Then $r^{m}-1=\left(r^{m / 2}-1\right)\left(r^{m / 2}+1\right)$. Now, by our assumption, $r^{m / 2} \equiv 1$ $(\bmod 4)$ so that $2 \mid r^{m / 2}+1$. That is, $2^{t-1}|| r^{m / 2}-1$. Let $K^{*}=\operatorname{GF}\left(r^{m / 2}\right)$. Let $\varphi: \epsilon \rightarrow \epsilon^{r m / 2}$ be the automorphism of $\widetilde{\mathbf{K}}$ of order 2 . Let $\operatorname{tr}: \mathbf{K}^{*} \rightarrow \mathbf{K}$ be the trace map. Then $\nu^{\varphi}=-\nu$ and $\nu^{2} \in K^{*}$. Let $W=K^{*} e_{1}+K^{*} e_{2}$.

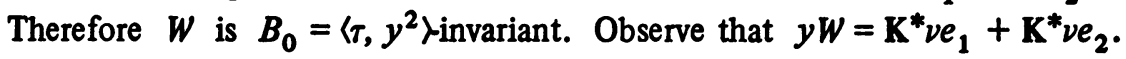
Let $\beta_{1}, \beta_{2}, \gamma_{1}, \gamma_{2} \in \mathrm{K}^{*}$. Then

$$
\begin{aligned}
g\left(\beta_{1} e_{1}+\beta_{2} e_{2}, \gamma_{1} \nu e_{1}+\gamma_{2} \nu e_{2}\right) & =\operatorname{Tr}\left(\beta_{1} \gamma_{2} \nu-\beta_{2} \gamma_{1} \nu\right) \\
& =\operatorname{tr}\left(\beta_{1} \gamma_{2}\left(\nu+\nu^{\varphi}\right)-\beta_{2} \gamma_{1}\left(\nu+\nu^{\varphi}\right)\right)=0
\end{aligned}
$$

since $\nu^{\varphi}=-\nu$. The spaces $W$ and $y W$ are orthogonal. Since $V$ is nonsingular, $W$ is nonsingular. We conclude that the $B_{0}$-module $W$ symplectically induces $V$. 
If $r \equiv 1(\bmod 4)$ then $s=0$ and $m=1$. So we may assume that $r \equiv$ $-1(\bmod 4)$. We must have $m=2$. Let $\varphi: \epsilon \rightarrow \epsilon^{r}$ be the automorphism of order two of $\widetilde{\mathbf{K}}=\operatorname{GF}\left(r^{2}\right)$. Then $\nu^{\varphi}=-\nu^{-1}$ since $2^{t-1} \mid \boldsymbol{V}+1$. Since $4 \mid r+1$ we may choose $\beta$ of order 4 so that $\beta^{\varphi}=\beta^{-1}=-\beta$. Let $W=$ $\left\{\gamma e_{1}+(\gamma \beta)^{\varphi} e_{2} \mid \gamma \in \widetilde{\mathbf{K}}\right\}$. Note that $W$ is a 2 -dimensional $\mathbf{K}$-subspace of $V$. Now

$$
y^{2}\left(\gamma e_{1}+(\gamma \beta)^{\varphi} e_{2}\right)=\nu^{2} \gamma e_{1}+\nu^{-2}(\gamma \beta)^{\varphi} e_{2}=\left(\nu^{2} \gamma\right) e_{1}+\left[\left(\nu^{2} \gamma\right) \beta\right]^{\varphi} e_{2} \in W .
$$

Also

$$
\tau y\left(\gamma e_{1}+(\gamma \beta)^{\varphi} e_{2}\right)=-\nu^{-1}(\gamma \beta)^{\varphi} e_{1}+\nu \gamma e_{2}=(\nu \gamma \beta)^{\varphi} e_{1}+\left[(\nu \gamma \beta)^{\varphi} \beta\right]^{\varphi} e_{2} \in W .
$$

So $W$ is $B_{0}=\left\langle\tau y, y^{2}\right\rangle$-invariant.

Observe that $y W=\left\{\gamma e_{1}-(\gamma \beta)^{\varphi} e_{2} \mid \gamma \in \widetilde{\mathbf{K}}\right\}$ since

$$
y\left(\gamma e_{1}+(\gamma \beta)^{\varphi} e_{2}\right)=(\nu \gamma) e_{1}+\nu^{-1}(\gamma \beta)^{\varphi} e_{2}=(\nu \gamma) e_{1}-(\nu \gamma \beta)^{\varphi} e_{2} .
$$

Therefore, $V_{B_{0}}=W+y W$ is a $\mathbf{K}\left[B_{0}\right]$-decomposition of $V$.

Let $\gamma e_{1}+(\gamma \beta)^{\varphi} e_{2} \in W$ and $\zeta e_{1}-(\zeta \beta)^{\varphi} e_{2} \in y W$. Then

$$
\begin{aligned}
g\left(\gamma e_{1}\right. & \left.+(\gamma \beta)^{\varphi} e_{2}, \zeta e_{1}-(\zeta \beta)^{\varphi} e_{2}\right)=\operatorname{Tr}\left(-\gamma(\zeta \beta)^{\varphi}-(\gamma \beta)^{\varphi} \zeta\right) \\
& =-\gamma(\zeta \beta)^{\varphi}-\gamma^{\varphi}(\zeta \beta)-(\gamma \beta)^{\varphi} \zeta-(\gamma \beta) \zeta^{\varphi} \\
& =-\gamma \zeta^{\varphi}\left(\beta^{\varphi}+\beta\right)-\gamma^{\varphi} \zeta\left(\beta^{\varphi}+\beta\right) \\
& =0 .
\end{aligned}
$$

So $W$ and $y W$ are orthogonal. Since $V$ is nonsingular, $W$ must also be nonsingular. Therefore, $V l_{B}$ is symplectically induced by the $\mathrm{K}\left[B_{0}\right]$-module $W$. The proof of (4.5) is now complete by (4.4).

(4.6) If $H \leqslant B$ of (2.4) then $H$ is not a 2-group.

This follows immediately from (3.22). Every proper subgroup of $S$ is reducible. Further, by (3.22) (3), $V l_{S}$ is symplectically induced from $K^{*}(1+\nu) e_{1}+K^{*}(1-\nu) e_{2}$ for the subgroup $\left\langle\tau, y^{2}\right\rangle \leqslant S$.

\section{REFERENCES}

1. T. R. Berger, Automorphisms of soluable groups, J. Algebra 27 (1973), 311-340.

2. - Hall-Higman type theorems. I, Canad. J. Math. 26 (1974), 513-531.

3. - Hall-Higman type theorems. III, Trans. Amer. Math. Soc. (to appear).

4. Hall-Higman type theorems. V, Pacific J. Math. (to appear).

5. - Hall-Higman type theorems. VIII, Proc. London Math. Soc. (to appear).

6. - Nilpotent fixed point free automorphisms groups of solvable groups, Math.

Z. 131 (1973), 305-312. 
7. T. R. Berger, Characters and derived length in groups of odd order, J. Algebra (to appear).

8. - Primitive solvable groups, J. Algebra 33 (1975), 9-21.

9. T. R. Berger and F. Gross, 2-length and the derived length of a Sylow 2-subgroup, Proc. London. Math. Soc. (to appear).

10. P. Hall and G. Higman, On the p-length of p-soluble groups and reduction theorems for Burnside's problem, Proc. London Math. Soc. 6 (1956), 1-42. MR 17, 344.

SCHOOL OF MATHEMATICS, UNIVERSITY OF MINNESOTA, MINNEAPOLIS, MINNESOTA 55455 